\title{
Joint Freehand Ultrasound and Endoscopic Reconstruction of Brain Tumors
}

\author{
Ruben Machucho-Cadena ${ }^{1}$, Sergio de la Cruz-Rodríguez ${ }^{2}$, \\ and Eduardo Bayro-Corrochano ${ }^{1}$ \\ ${ }^{1}$ CINVESTAV, Unidad Guadalajara, Departamento de Ingeniería Eléctrica y \\ Ciencias de la Computación, Jalisco, México \\ $\{$ rmachuch, edb\}@gdl. cinvestav . mx \\ 2 Instituto Superior Politécnico "José A. Echeverría", Havana, Cuba \\ sergio@electrica.cujae.edu.cu
}

\begin{abstract}
We present an approach for reconstructing the 3D shape of brain tumors for applications in neurosurgery from 2D ultrasound (US) images. We record simultaneously endoscopic and ultrasonic images, and the pose of the endoscopic camera by an optical tracking system. The $3 \mathrm{D}$ pose of the ultrasound probe is determined by tracking the $2 \mathrm{D}$ position of the ultrasound probe in successive endoscopic images by image processing techniques (Hough Transform, Particle Filtering) and finally the $3 \mathrm{D}$ position is computed by the known camera geometry and multiple view geometry using conformal geometric algebra. When the 3D US probe position is calculated, we compound multiple 2D US images into a $3 \mathrm{D}$ volume.
\end{abstract}

Keywords: endo-neuro-sonography, 3D reconstruction, brain tumor segmentation.

\section{Introduction}

Recent trends in minimally invasive brain surgery aim at the use of the joint acquisition of endoscopic and ultrasound (US) images, a technique that has been called endo-neuro-sonography (ENS) 3]. This is easily understandable. Endoscopic images are an essential component of minimally invasive techniques since without them the surgeons can not see what they are doing. However, unlike magnetic resonance (MR), tomographic (CAT) and ultrasound images, endoscopic images cannot see beyond opaque structures. So it is desirable to also have one of those imaging techniques, been the ultrasound the most suitable of them for intraoperative scenarios.

Now that we can jointly record endoscopic and ultrasonic images it would be desirable to integrate them into a $3 \mathrm{D}$ model that can help surgeons to better locate brain structures such as tumors during the course of the operation. The scientific community has paid significant attention to this goal in recent years 812. Virtually all of the available methods use either a magnetic or optic tracking system (and even a combination of both) to locate the tip of the US 
probe in 3D space. These systems are sometimes difficult to implement in intraoperative scenarios. It is known that the ferromagnetic properties of surgical instruments (among others) can affect magnetic tracking systems [5]. On the other hand, the classic optical tracking approach of putting markers on the US probe is useless in many modern systems where the US probe is flexible and rotates around its axis.

Unlike methods reported in related work, we eliminate the need of an independent opto-magnetic tracking system because we calculate the pose (i.e. the position and orientation) of the US probe using multiple view geometry in successive endoscopic images. To the best of our knowledge, this approach has not been reported before. The whole process can be summarized as follows:

- We have a setup (Sect. 2) that consists of an Aloka ENS equipment and a Polaris stereo tracking system that we use to visually track the endoscopic camera. The US probe is almost always seen in the endoscopic images.

- So we track the US probe (Sect. 3) in the endoscopic images using two independent tracking methods: Hough transform and particle filters. Here we estimate the orientation of the US probe axis and the $2 \mathrm{D}$ location of the tip of the probe.

- We segment the relevant structures in the US images (Sect. 4) applying image processing methods (roi, thresholding, morphological filters).

- With the information about the US probe in successive images we use multiple view methods [4] with conformal geometric algebra to calculate the 3D pose of the probe (Sect. 5 ).

- The relevant structures are represented in a 3D visual environment in their corresponding locations.

\section{System Setup}

Figure 1 shows the experimental setup. The equipment setup is as follows: The Aloka ENS equipment provides an US probe that is introduced through a channel in the endoscope (Fig. 2a) and is seen by the endoscope. We also employ a Polaris visual tracking system by which we calculate the $3 \mathrm{D}$ position of the endoscopic camera. The camera was calibrated using the method described in 11 .

The US probe is flexible and is rotating around its longitudinal axis at about 60 rpms. It can also move back and forth and since the channel is wider than the probe there is also a random movement around the channel. The US image is orthogonal to the US probe axis (Fig. 2b). We know that in one small interval of time $x$, the ultrasound probe is fixed, and the endoscopic camera undergoes a movement, which is equivalent to an inverse motion, i.e. the endoscopic camera is fixed, and ultrasound probe undergoes a movement.

\section{Tracking the Ultrasound Probe in Endoscopic Images}

To track the US probe that is seen in endoscopic images we use two alternative techniques: Generalized Hough Transform with AE, and a Particle Filter. We 


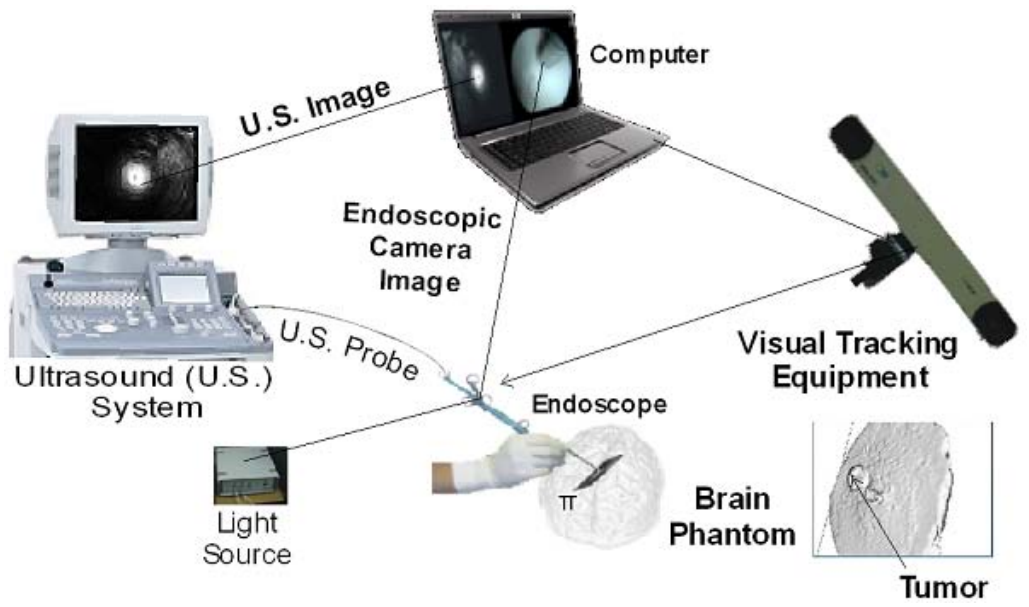

Fig. 1. Experimental Setup

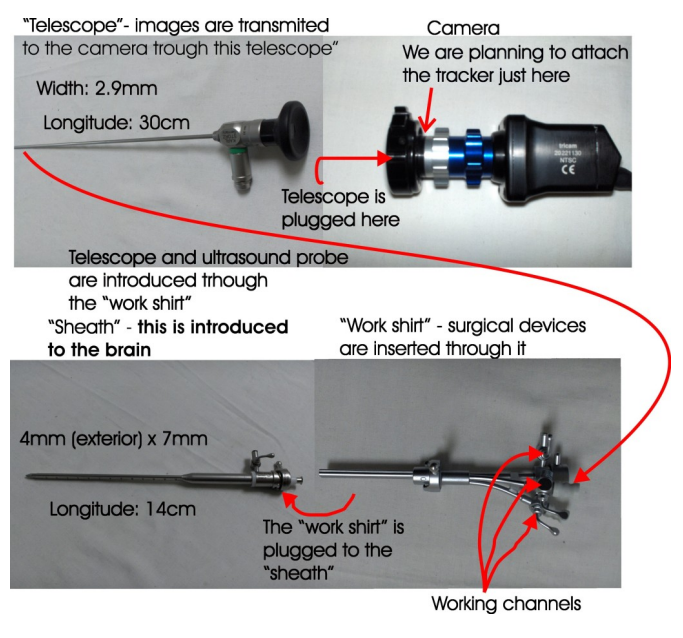

(a)

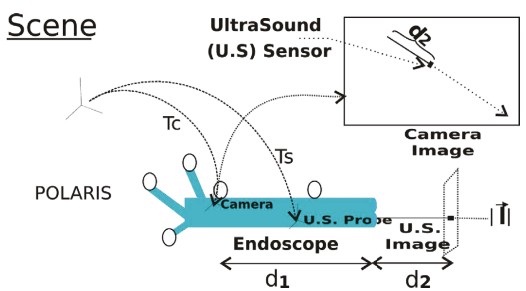

(b)

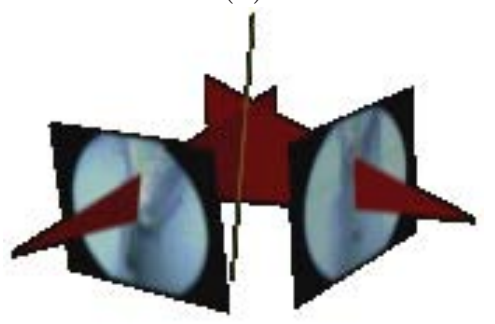

(c)

Fig. 2. System setup. a) ENS equipment. b) Diagram of the scene. The US image is orthogonal to the US longitudinal axis. c) A sample of intersection of planes.

track the axis line of the US probe throughout the images. This line allows us to compute its $3 \mathrm{D}$ coordinates using the camera projection matrix. Such projection is done as follows: every line is backprojected to form a plane in the space, which contains the line and the camera center. Using two consecutive images, the intersection of its respective planes yields the line in 3D. To obtain a more accurate result, we take only the unit vector of this line because the calculations of the translation in the projection matrix is up to a scalar factor. We translate 
the first point (the tip of the endoscope) obtained by the Polaris lecture by a distance $d_{2}$ (Fig. 2 b) along the direction of the unit vector in the direction of the previously obtained line, and this translated point corresponds to the position of the US sensor in $3 \mathrm{D}$, making possible to obtain the plane $\Pi$ to make the virtual representation. Now we give a brief overview of the methods that were independently used to track the axis of the US probe.

\subsection{The Generalized Hough Transform Tracker}

The Generalized Hough Transform (GHT) 2] is used to find an object in an image using a template. The matching process is based in a voting system for the possible orientations, positions and also scalings of the template. The votes are stored in an accumulator and the location in the accumulator with the greatest amount of votes will be the winner. This tracking method is robust to occlusion and illumination changes. We use it to get a first good approximation of the template in the image. To start, we need to make a R-table which completely describes the template.. We select a reference point $\left(x_{r e f}, y_{r e f}\right)$, and draw a line to any point of the boundary. Then we compute the distance and the direction from that point to the reference point, i.e. $(r, \beta)$. The orientation of the boundary $\Omega_{i}$ will be the index to the $(r, \beta)$ values. To apply the GHT algorithm to the endoscopic images, a Gauss pyramid was previously applied to reduce the size of the image and its edges were obtained using a Canny filter.

An analysis of the orientation of the edges (AE) in the image was made in order to increase the accuracy of the GHT tracking algorithm. So we have two estimated lines (the first line obtained using GHT and second line obtained using $\mathrm{AE})$, and these are weighted to obtain a total estimated line (EL).

$$
E L=0.65 \times G H T+0.35 \times A E
$$

\subsection{The Particle Filter Tracker}

Another approach to perform visual tracking is to use a Bayesian tracker, treating motion tracking as a Bayesian state estimation problem. The most widely used Bayesian tracker is the Kalman filter. However Kalman filters require a Gaussian observation probability and a Gaussian posterior probability density. Our observations show that the random movement of the ultrasound probe can hardly be described as Gaussian. This situation made us look for a more general tracker: the particle filter. Particle filters emerged from the pioneer work of Isard and Blake [6]. The reader is referred there for further information.

To estimate the US probe axis we define its state vector $s^{(i)}=\left[d_{x}^{(i)}, d_{y}^{(i)}, d_{\theta}^{(i)}\right]^{\top}$. This vector encodes the US 2D pose as a variation in position and rotation of a candidate line with respect to an initial line estimate. This model evolves in each stage according to

$$
S_{t}=S_{t-1}+N_{t}
$$

with $N_{t}$ been white Gaussian noise. 
To obtain the weight of each candidate line, a support area of the initial line estimate is selected and is then translated and rotated according to the state vector of the candidate line. Both the image of the support area around the initial line in the reference image and the image corresponding to the transformed support area in the current image are compared by means of the Bhattacharyya distance between their color histograms as described in [7.

\subsection{Tracking Results}

The GHT method was applied as explained and the particle filter was applied using up to $N=300$ particles but little difference is observed in the results when $N>$ 100. Several images and sequences has been tested. Figure 3 shows some results on four images for each method. The results for the GHT method are shown in Fig. 3 a while the results for the particle filter method are shown in Fig. 3 b.

Both tracking methods performed well in practice. However, we prefer the GHT method (Sect. 3.1) because is faster and also because the particle filter method is non-deterministic.
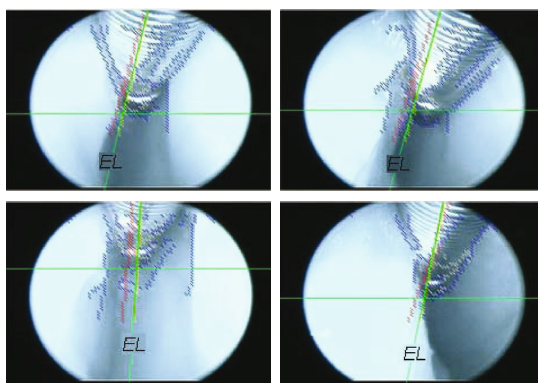

(a)

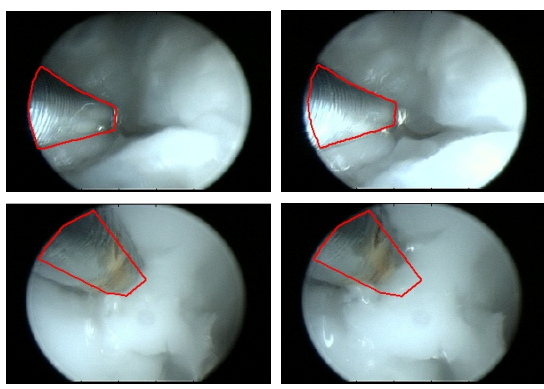

(b)

Fig. 3. Some tracking results using our ENS equipment in a brain phantom. a) The results for the GHT method. b) The results for the particle filter method.

\section{Ultrasound Image Processing}

The goal in this stage is to segment interesting structures such as tumors in brain images. We use morphological operators to fill small holes that appear due to the subsampling algorithm of the Aloka ultrasound system. The closing morphological operator of the image $I$ with the subimage $M$ (structuring element) is defined $I \cdot M=(I \oplus M) \ominus M$ where $\oplus$ and $\ominus$ represent the dilatation and erosion morphological operators, respectively [2].

We process the ultrasound images in the following way:

- We take a copy of the original image and select a region of interest (ROI) that can be the whole image if desired. The ROI will be the same for all images. 
- We eliminate from the ROI the central part of the image if present. We exclude that part because it only contains noise.

- Apply a threshold to the gray levels of the ROI, to select only the highest levels.

- Apply closing morphological operator to fill the holes of the ROI.

- Use the chain code to calculate the smallest areas of the ROI, and eliminate them.

- Apply logical AND operation between the ROI and the original image. The result is the segmented tumor, which is to be represented in $3 \mathrm{D}$.

Figure 4 shows an example of the segmented tumor.

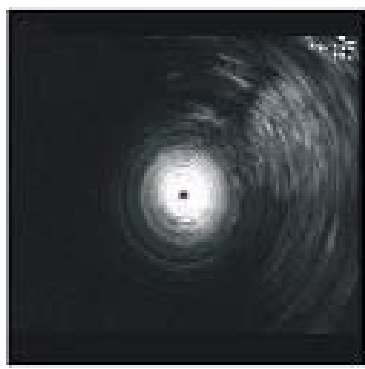

(a)

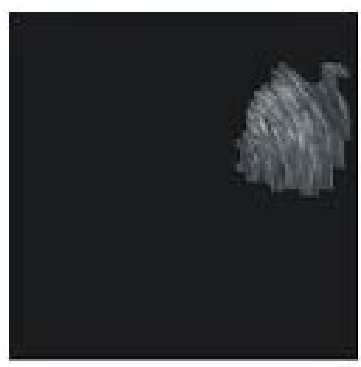

(b)

Fig. 4. Isolating the tumor. a) Original US image to be segmented. b) ROI after applying morphological operator.

\section{Calculating the 3D Pose of the Tumor}

Conformal Geometric Algebra (CGA) represents geometric entities as points, lines, planes, spheres, pair of points, dilators, etc. in economical and compact form. It has great potential for applications in medicine, artificial vision and robotics [1. The CGA preserves the Euclidean metric and adds two basis vectors: $\mathrm{e}_{+}, \mathrm{e}_{-}\left(\right.$where $\mathrm{e}_{+}{ }^{2}=1$ and $\left.\mathrm{e}_{-}{ }^{2}=-1\right)$, which are used to define the point at the origin $\mathrm{e}_{0}=\frac{1}{2}\left(\mathrm{e}_{-}-\mathrm{e}_{+}\right)$and the point at the infinite $\mathrm{e}=\mathrm{e}_{-}+\mathrm{e}_{+}$. The points in CGA are related with the Euclidean space by: $\underline{p}=\mathbf{p}+\frac{\mathbf{p}^{2}}{2} \mathrm{e}+\mathrm{e}_{0}$. A sphere in dual form is represented as the wedge of four conformal points that lies on sphere $\underline{s}^{*}=\underline{a} \wedge \underline{b} \wedge \underline{c} \wedge \underline{d}$, its radius $\rho$ and its center $\underline{p}$ in $\mathcal{R}^{3}$ can be obtained using: $\rho^{2}=\frac{\underline{s}^{2}}{(\underline{s} \cdot e)^{2}}, \underline{p}=\frac{\underline{s}}{-(\underline{s} \cdot e)}+\frac{1}{2} \rho^{2} \mathrm{e}$. A plane in dual form is defined as a sphere, but the last point is at the infinity: $\underline{\pi}^{*}=\underline{a} \wedge \underline{b} \wedge \underline{c} \wedge$ e. A line in dual form is represented as the wedge of two points and the infinity point: $\underline{L}^{*}=\underline{a} \wedge \underline{b} \wedge \mathrm{e}$.. A line can also be calculated as the intersection of two planes: $\underline{L}=\underline{\pi_{1}} \wedge \underline{\pi_{2}}$. This equation is used to calculate the $3 \mathrm{D}$ line that represents the ultrasound probe axis; as we already mentioned, we are only taking the unit vector of this line. To achieve a translation by a distance $d_{2}$ from a point $\underline{p_{1}}$ in the direction of a line and to obtain $\underline{p_{2}}: T=\exp \left(\frac{1}{2} d_{2} L\right), \underline{p_{2}}=T \underline{p_{1}} \widetilde{T}$. 
The last equation is used to find the position of the ultrasound sensor in order to put the segmented ultrasound image in $3 \mathrm{D}$ space; where $p_{1}$ represents the tip of the endoscope obtained by the Polaris tracking system. $\overline{\text { Figure }}[\mathbf{5}$ a shows an example.

Further results are presented in Fig. $5 \mathrm{~b}-\mathrm{c}$. The 3D model was built by stacking several estimations and finding the convex hull of their $3 \mathrm{D}$ space [10].

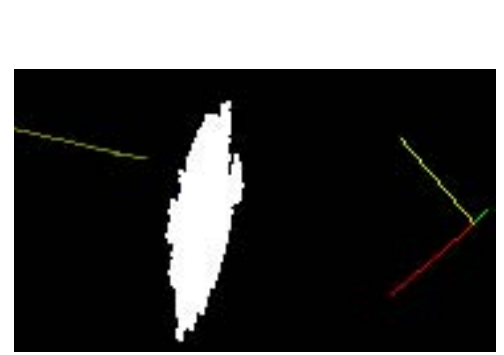

(a)

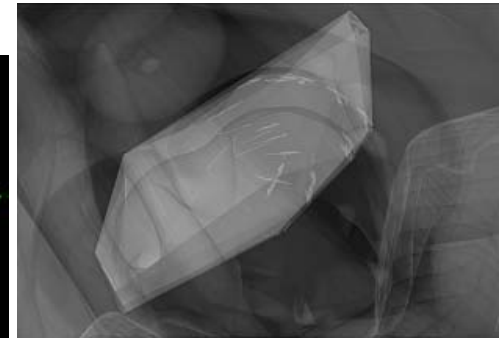

(b)

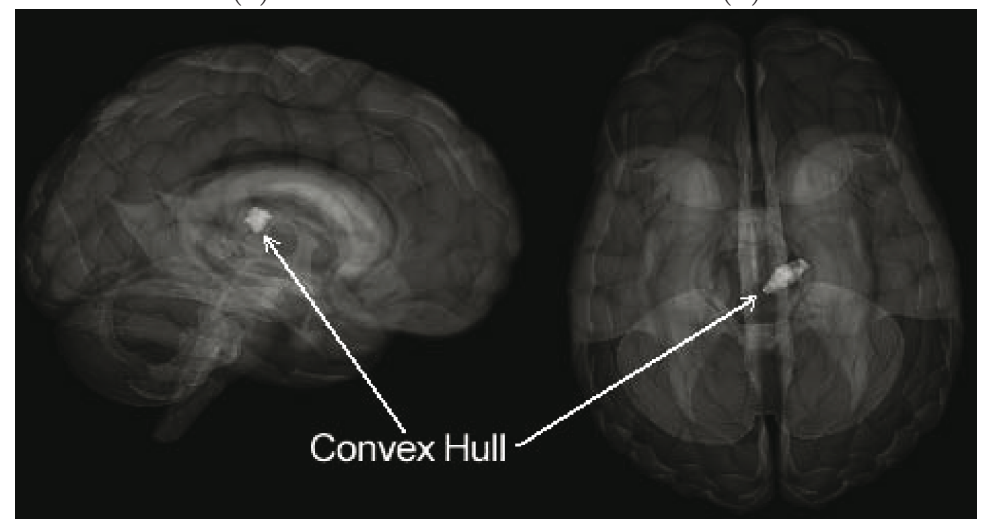

(c)

Fig. 5. Results of the 3D reconstruction process. a) Ultrasound probe axis, the segmented tumor and the Polaris reference frame. b) Convex hull of the segmented tumor in $3 \mathrm{D} . \mathrm{c})$ The tumor in a brain phantom model.

\section{Conclusions}

We have addressed the problem of obtaining 3D information from joint ultrasound and endoscopic images obtained with ENS equipment. In order to register both sources we developed two alternative methods to locate the US probe tip in endoscopic images, one of them using Generalized Hough Transform and further refinement through of an analysis of the orientation edges in the image and the other one using a particle filter. Some preliminary results are showed. As for the ultrasound image, we presented a method to segment interesting brain structures. We use a novel approach to find the 3D position of the US images 
using multiple view geometry with conformal geometric algebra. Results are showed in 3D space, the 3D information been calculated from the results obtained by the tracking process in endoscopic images. The performance of the proposed approach has been demonstrated using several images where occlusion and changes in illumination and contrast are present. Results indicate that the proposed approach is robust.

\section{References}

1. Bayro Corrochano, E.: Geometric Computing for Perception Action Systems: Concepts, Algorithms, and Scientific Applications. Springer, Heidelberg (2001)

2. Castleman, K.R.: Digital Image Processing. Prentice Hall, Englewood Cliffs (1996)

3. Resch, K.D.M.: Transendoscopic Ultrasound for Neurosurgery. Springer, Heidelberg (2006)

4. Hartley, R.I., Zisserman, A.: Multiple View Geometry in Computer Vision. Cambridge University Press, Cambridge (2004)

5. Hummel, J.B., et al.: Design and Application of an Assessment Protocol for Electromagnetic Tracking Systems. Medical Physics 32, 2371-2379 (2005)

6. Isard, M., Blake, A.: Condensation - Conditional Density Propagation for Visual Tracking. Int. J. Comput. Vis. 29(1), 5-28 (1998)

7. Nummiaro, K., Koller-Meier, E., Van Gool, L.: An Adaptive Color-based Particle Filter. Image Vis. Comput. 21(1), 99-110 (2003)

8. Unsgaard, G., et al.: Intra-operative 3D Ultrasound in Neurosurgery. Acta Neurochir(Wien) 148(3), 235-253 (2006)

9. Zhang, Z.: A Flexible New Technique for Camera Calibration. J. IEEE Transactions on Pattern Analysis and Machine Inteligence 22(11), 1330-1334 (2000)

10. Barber, C., et al.: The Quickhull algorithm for convex hulls. ACM Trans. on Mathematical Software 22(4), 469-483 (1996), http://www.qhull.org

11. Bayro Corrochano, E., Daniilidis, K.: The Dual Quaternion Approach to Hand-Eye Calibration. In: International Conference on Pattern Recognition (ICPR 1996), Vienna, Austria, vol. 1, pp. 318-322. IEEE Computer Society Press, Los Alamitos (1996)

12. Feuerstein, M., et al.: Magneto-Optic Tracking of a Flexible Laparoscopic Ultrasound Transducer for Laparoscope Augmentation. In: Ayache, N., Ourselin, S., Maeder, A. (eds.) MICCAI 2007, Part I. LNCS, vol. 4791, pp. 458-466. Springer, Heidelberg (2007) 